\title{
Engineering Saccharomyces cerevisiae for the production of the valuable monoterpene ester geranyl acetate
}

Tao $\mathrm{Wu}^{1,2,3 \dagger}$, Siwei $\mathrm{Li}^{2,3 \dagger}$, Bolin Zhang ${ }^{1 *}$, Changhao $\mathrm{Bi}^{2,3^{*}} \oplus$ and Xueli Zhang ${ }^{2,3^{*}}$

\begin{abstract}
Background: Geranyl acetate is widely used in the fragrance and cosmetic industries, and thus has great economic value. However, plants naturally produce a mixture of hundreds of esters, and geranyl acetate is usually only present in trace amounts, which makes its economical extraction from plant sources practically impossible. As an ideal host for heterologous production of fragrance compound, the Saccharomyces cerevisiae has never been engineered to produce the esters, such as geranyl acetate.
\end{abstract}

Results: In this study, a heterologous geranyl acetate synthesis pathway was constructed in S. cerevisiae for the first time, and a titer of $0.63 \mathrm{mg} / \mathrm{L}$ geranyl acetate was achieved. By expressing an Erg20 mutant to divert carbon flux from FPP to GPP, the geranyl acetate production increased to $2.64 \mathrm{mg} / \mathrm{L}$. However, the expression of heterologous GPP had limited effect. The highest production of $13.27 \mathrm{mg} / \mathrm{L}$ geranyl acetate was achieved by additional integration and expression of tHMG1, IDI1 and MAF1. Furthermore, through optimizing fermentation conditions, the geranyl acetate titer increased to $22.49 \mathrm{mg} / \mathrm{L}$.

Conclusions: We constructed a monoterpene ester producing cell factory in S. cerevisiae for the first time, and demonstrated the great potential of this system for the heterologous production of a large group of economically important fragrance compounds.

Keywords: Monoterpene, Geranyl acetate, ERG20, tHMG1, IDI1, MAF1

\section{Background}

Monoterpenes constitute a subclass of terpenoids [1] that are widely used as additives in the food, pharmaceutical, agrichemical and cosmetic industries, due to their strong flavor, fragrance and physiological activity [2, 3]. Moreover, some monoterpenes were shown to have great potential as biofuels [4], which prompted increased attention from the research community in recent years. The basic scaffold of monoterpenes contains two isoprene units that are biosynthesized from geranyl diphosphate (GPP),

\footnotetext{
*Correspondence: Zhangbolin888@sina.com.cn; bi_ch@tib.cas.cn; zhang_xl@tib.cas.cn

${ }^{\dagger}$ Tao Wu and Siwei Li have contributed equally to this work

${ }^{1}$ College of Biological Sciences and Technology, Beijing Forestry

University, Beijing 100083, People's Republic of China

${ }^{2}$ Tianjin Institute of Industrial Biotechnology, Chinese Academy

of Sciences, Tianjin 300308, People's Republic of China

Full list of author information is available at the end of the article
}

which in turn is synthesized from isopentenyl diphosphate (IPP) and its isomer dimethylallyl diphosphate (DMAPP), derived from either the mevalonate (MVA) pathway or the 2C-methyl-D-erythrtiol 4-phosphate (MEP) pathway $[5,6]$. As the precursor of monoterpenes such as geraniol and linalool, GPP reacts with one more IPP and gives rise to farnesyl diphosphate (FPP) [7, 8], which is the precursor of sesquiterpenes, squalene, diterpenes, GGPP and so on.

Monoterpenes are mainly produced by plants, albeit at extremely low concentrations [9], and the traditional chemical synthesis and bio-extraction processes are both costly and environmentally harmful. However, a number of research groups have been able to produce natural products by metabolic engineering of microbial cell factories [10,11], most often derived from the model organisms Escherichia coli and Saccharomyces cerevisiae 
[12-14]. The production of monoterpenes by metabolic engineering has also been reported, but most of the cases involved relative low production, which hindered their industrial application [9]. Previous work has shown that engineered $E$. coli could produce $400 \mathrm{mg} / \mathrm{L}$ of limonene and approximately $100 \mathrm{mg} / \mathrm{L}$ of perillyl alcohol [13], and engineered yeasts were able to produce $95 \mu \mathrm{g} / \mathrm{L}$ of linalool [15], as well as $36.04 \mathrm{mg} / \mathrm{L}-2.0 \mathrm{~g} / \mathrm{L}$ of geraniol [16, 17].

Saccharomyces cerevisiae possesses a native MVA pathway [6], which makes it suitable for the synthesis of monoterpenes. Some reports also demonstrated the potential of metabolic engineering for monoterpene production in S. cerevisiae [18]. GPP is synthesized by the bifunctional enzyme ERG20 (Fig. 1), which has both GPP synthase (GPPS) and farnesyl pyrophosphate synthase (FPPS) activities [19]. To decrease the metabolic flux towards FPP, mutants with changes in the FPP synthesis domain of Erg20p were screened, and mutations at position 197 (K197G, C, S, T, D, E) [18] and a double mutant (N127W-F96W) [20] were proved to dramatically increase the yield of monoterpenes. To increase the production of sabinene, the sabinene synthase SpSabS1 was fused to the Erg20 variant, and $1.87 \mathrm{mg} / \mathrm{L}$ sabinene production was achieved, which represents a 3.5-fold increase compared with that achieved via the separate expression of Erg20 and SpSabS1 [20]. By the screening of different sources of GESs and GPPSs, as well as fusions of the two proteins, Hou' group achieved the titer of $293 \mathrm{mg} / \mathrm{L}$ geraniol in S. cerevisiae [21]. Furthermore, dynamic control of ERG20 expression combined with minimized endogenous downstream metabolism led to impressive progress in the production of geraniol [22].

On the other hand, HMG-CoA reductase was identified as a key rate-limiting enzyme in the MVA pathway of S. cerevisiae [23, 24], and a truncated 3-hydroxyl3 -methylglutaryl-CoA reductase gene (tHMGR) was overexpressed to increase the supply of mevalonate. DMAPP is the common substrate for the biosynthesis of both GPP and tRNA, and MAF1 represses the transcriptional activity of RNA polymerase III, serving as a negative regulator in the biosynthesis of tRNA [16]. The isoprenoid diphosphate isomerase IDI1 catalyzes the interconversion between DMAPP and IPP [25], but the formation of one molecule of GPP requires two molecules of IPP and one molecule of DMAPP. Since the ratio of IPP to DMAPP is not optimal for GPP biosynthesis, Jingwen Zhou's research indicated that the

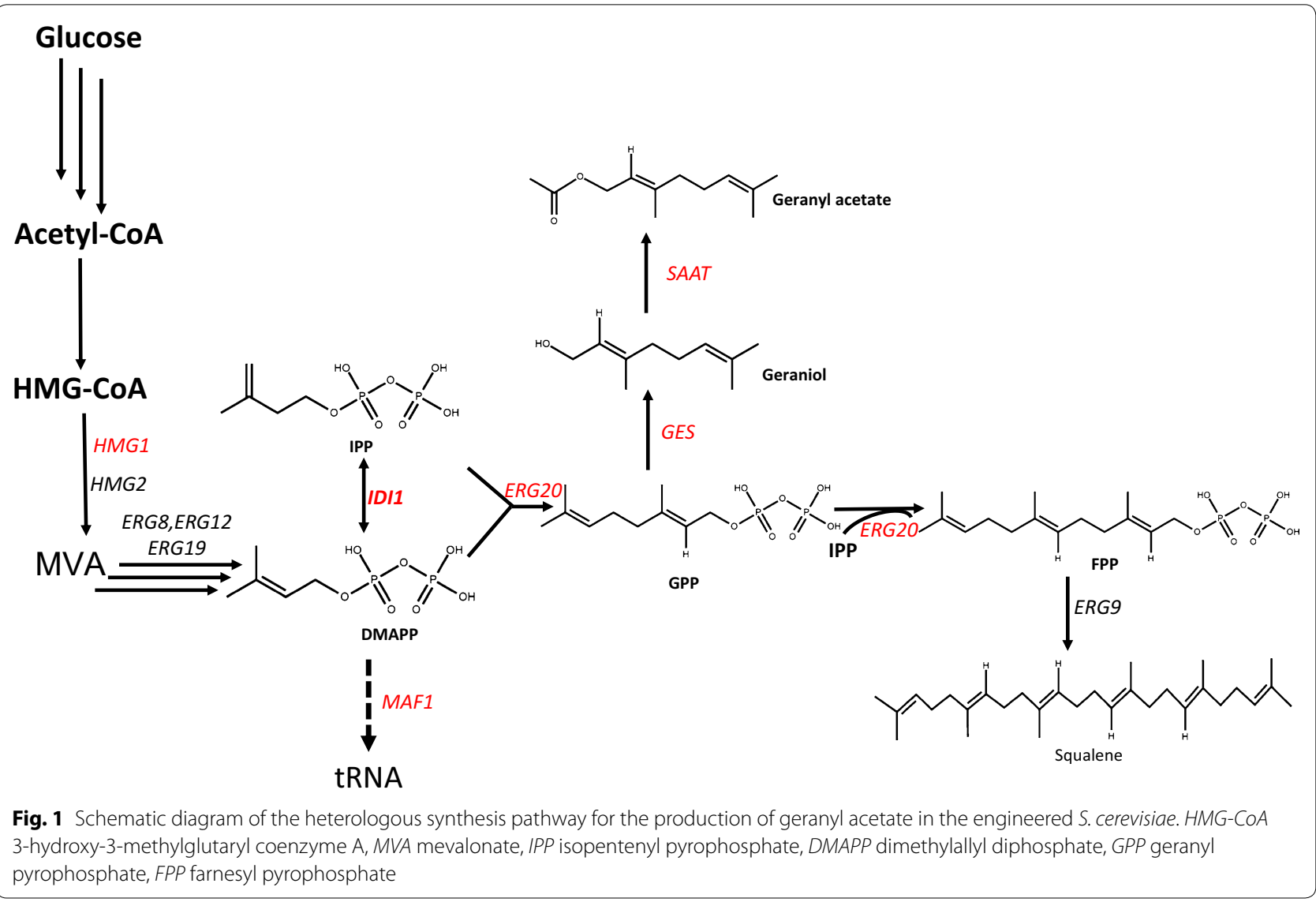


isomerase IDI1 was the rate-limiting enzyme in geraniol production [16]. Thus, overexpression of truncated HMG-CoA (tHMG1), IDI1 and MAF1 could improve the production of monoterpenes.

Geranyl acetate, an acyclic monoterpene ester derived from geraniol, is widely used in the cosmetics industry due to its pleasant scent, and it was also recently discovered to have an antinociceptive activity [26], making it a compound with great economic value. However, plants naturally produce a mixture of hundreds of esters, and geranyl acetate only makes up a small percentage of the total, which makes its extraction and traditional plant-based production uneconomical [27, 28]. Albeit the great commercial potential, as far as we know, no research has focused to heterologous produce ester fragrance compounds. And as an ideal host for heterologous production of fragrance compound, the $S$. cerevisiae has never been engineered to produce the esters, such as geranyl acetate.

Thus, in this study, we intended to construct a S. cerevisiae cell factory for production of geranyl acetate to study and demonstrate the capacity of this system for heterologous production of this group of economically important fragrance compounds, and explore the fermentation methods and conditions for the production process (Fig. 1). Geraniol synthase (GES) [16, 22, 29] from Ocimum basilicum and alcohol acyltransferases (AATs) [29] from strawberry (Fragaria $\times$ ananassa) (SAAT) were introduced into the yeast chromosome. Several groups have used the GES from $O$. basilicum for the formation of geraniol, and SAAT showed a high affinity and efficiency for the biosynthesis of geranyl acetate [29].

\section{Methods}

\section{Media, strains and plasmids}

Escherichia coli Trans T1 (TransGen, China) was used as the host for plasmid construction and amplification. The cells were grown at $37{ }^{\circ} \mathrm{C}$ in Luria-Bertani (LB) medium $(1 \% \mathrm{NaCl}, 1 \%$ tryptone and $0.5 \%$ yeast extract with $100 \mathrm{mg} / \mathrm{L}$ of ampicillin (Solarbio, China). S. cerevisiae

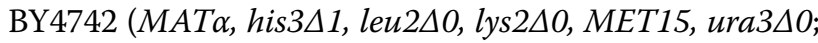
Euroscarf, Germany) was used as the host strain for DNA assembly and integration. The cells were cultivated at $30{ }^{\circ} \mathrm{C}$ in yeast-extract peptone dextrose (YPD) medium ( $2 \%$ glucose, $2 \%$ peptone, and $1 \%$ yeast extract). Synthetic complete drop-out medium lacking leucine and/or histidine (SC-LEU, SC-LEU-HIS, SC-LEU-HIS-TRP) was used for transformant selection. For solid media, $2 \%$ agar was added.

The codon-optimized genes encoding geraniol synthase (GES) from Ocimum basilicum without the $\mathrm{N}$-terminal transit peptide (1-34aa), and alcohol acyltransferases (AATs) (SAAT) from cultivated strawberry (Fragaria $\times$ ananassa) were synthesized by GENEWIZ (Suzhou, China) and embedded in plasmid pUC57Amp. The sequences of the two genes are provided in Additional file 1. The SAAT and GES genes were excised from pUC57-SAAT/GES with SexAI and AscI. $S A A T$ was ligated into the vector pM2 under the control of the $P G K 1$ promoter and $A D H 1$ terminator, and pM3-GES was constructed with GES under the control of the TEF2 promoter and CYC1 terminator. $t H M G 1$ containing the catalytic domain of HMG1 was amplified from the genome of $S$. cerevisiae using the primer pair SexA-tHMG1/AscI-tHMG1 and cloned into vector pM4 using T4 ligase (Thermo, USA). The plasmids pM4ERG20(F96W-N127W), pM2-MAF1 and pM3-IDI1 were constructed in the same way (Table 1).

Table 1 Strains and plasmids used in this study

\begin{tabular}{|c|c|c|}
\hline Name & Description & Source \\
\hline pUC57-GES & Cloning vector with a synthetic version of the GES gene from 0 . basilicum & GENEWIZ \\
\hline pUC57-SAAT & $\begin{array}{l}\text { Cloning vector with a synthetic version of the SAAT gene from cultivated strawberry (Fra- } \\
\text { garia } \times \text { ananassa) }\end{array}$ & GENEWIZ \\
\hline pEASY-Blunt & Cloning vector for blunt ligation & This study \\
\hline pM3-GES & pEASY-Blunt vector with pTEF2-GES-tCYC1 cassette & This study \\
\hline pM2-SAAT & pEASY-Blunt vector with pPGK1-SAAT-tADH1 cassette & This study \\
\hline pM4-ERG20(F96W-N127W) & pEASY-Blunt vector with pTDH3-ERG20(F96W-N127W)-tTPI1 cassette & This study \\
\hline pM2-MAF1 & pEASY-Blunt vector with pPGK1-MAF1-tADH1 cassette & This study \\
\hline pM3-IDI1 & pEASY-Blunt vector with pTEF2-IDI1-tCYC1 cassette & This study \\
\hline pM4-tHMG1 & pEASY-Blunt vector with pTDH3-tHMG1-tTPI1 cassette & This study \\
\hline pRS313-TRP & pRS313 vector: HIS selection marker was replased withTRP & This study \\
\hline pRS313-GPPS ${ }_{\text {At }}$ & pRS313-TRP vector with pPGK-GPPS ${ }_{A t}-\mathrm{tCYC1}$ cassette & This study \\
\hline pRS313-GPPS & pRS313-TRP vector with pPGK-GPPS ${ }_{M p}$-tCYC1 cassette & This study \\
\hline
\end{tabular}


The plant GPP synthase genes were amplified from cDNA of Arabidopsis thaliana and Mentha piperita, and cloned into the expression vector pRS313-TRP using T4 ligase. The maps of the corresponding expres-

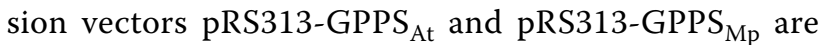
shown in Additional file 1: Fig. S1. All plasmids were verified by PCR and DNA sequencing, and the relevant primers are listed in Additional file 1: Table S1.

\section{Site-directed Mutagenesis of the farnesyl diphosphate synthase ERG20}

Two point-mutations (F96W and N127W) were introduced into ERG20 by overlap-extension PCR. Yeast genomic DNA was used as the template, and the ERG20 gene was divided into three parts, 1-96aa, 94-127aa and 127-353aa. The three parts of the gene were amplified separately using primers with embedded mutations, and then fused into a complete mutated gene by overlap-extension PCR. The corresponding primers are listed in Additional file 1: Table S1.

\section{Genetic manipulation of S. cerevisiae}

The construction and assembly of functional transcriptional units on the chromosome of S. cerevisiae was performed by the DNA assembler method [30,31]. The transcription unit encoding GES ( $p T E F 2-G E S-t C Y C 1$ ) was amplified by PCR from plasmid pM2-GES, that of SAAT ( $p P G K 1-G E S-t A D H 1)$ from pM3-SAAT, and that of ERG20 ( $p T D H 3-E R G 20(F 96 W-N 127 W)-t T P I 1)$ from pM4-ERG20. The selection marker and integration locus fragments were amplified by PCR. All primers used for amplification and integration into the S. cerevisiae chromosome are listed in Additional file 1: Table S1. Strain GA01 was constructed by integrating the GES and SAAT cassettes into the gal 80 site along with the LEU selection marker, the strain GA02 was constructed by integrating the GES, SAAT and ERG20(F96W-N127W) cassettes into the gal 80 site along with the LEU selection maker (Fig. 2), and strain GA03 was constructed by integrating the tHMG1, MAF1 and IDI1 cassettes into the NDT80 site of strain GA02 along with the HIS selection maker.

The DNA fragments, GPP synthase expression plasmid pRS313-GPPSAt and pRS313-GPPSMp were all

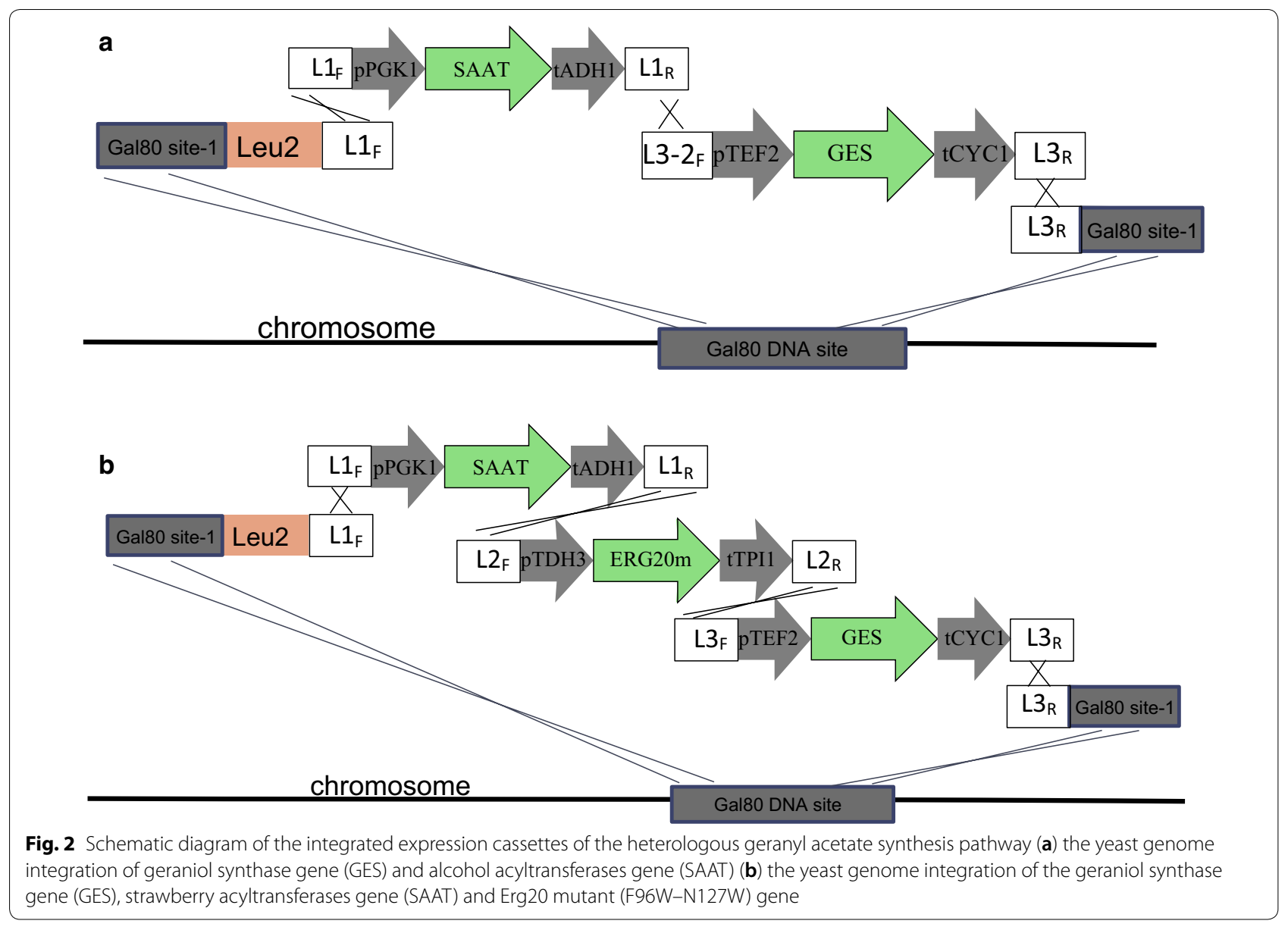


introduced into S. cerevisiae BY4742 by conventional electroporation method. When four or five fragments (each fragment was $100 \mathrm{ng}$ ) were used for homologous recombination (HR) in S. cerevisiae, about 200-400 colonies could be achieved.

\section{Yeast cultivation and PCR confirmation}

For PCR confirmation of transformants, single colonies were used to inoculate $4 \mathrm{~mL}$ of SC-LEU/SC-LEU-HIS/ SC-LEU-HIS-TRP medium, and grown at $30^{\circ} \mathrm{C}$ and $250 \mathrm{rpm}$ overnight. Cells were harvested by centrifugation, and the genomic DNA was extracted using the Yeast Gene DNA Kit (CW Biotech, China). $2 \mu \mathrm{L}$ of total DNA was used as template for PCR using the $2 \times$ Taq Master Mix (CW Biotech). 10 colonies from SC-Leu agar plates were randomly picked and inoculated in SC-Leu medium. After that, genomic DNA was extracted, and PCR determination was performed respectively. The ratio of positive clones to all the colonies was calculated to be above $90 \%$.

\section{Cell-culture, extraction and quantification of geranyl acetate}

The correct colonies were picked and grown in the corresponding synthetic complete drop-out medium or YPD medium overnight, transferred into a flask with fresh medium to yield an initial $\mathrm{OD}_{600}$ of $0.05-0.10$, and cultured for 6 days at $30^{\circ} \mathrm{C}$ and $250 \mathrm{rpm}$. The increase of cell biomass during the fermentation process was detected by measuring the $\mathrm{OD}_{600}$ value using a UV-2550 spectrophotometer (Shimadzu, Japan).

To quantify the titer of geranyl acetate in the yeast cultures, aliquots comprising $1 \mathrm{~mL}$ of the fermentation broth were concentrated by centrifugation at $16,200 \times g$ for $2 \mathrm{~min}$, after which $1 \mathrm{~mL}$ of $n$-hexane was added to extract the products that were secreted into the medium. The cell pellet was extracted with another $1 \mathrm{~mL}$ of $n$-hexane under ultrasonic agitation for $30 \mathrm{~min}$, and the n-hexane phase was collected by centrifugation at $16,200 \times g$ for $2 \mathrm{~min}$. The two extraction liquids were mixed, and $1 \mu \mathrm{L}$ of the combined extract was analyzed using a Agilent 5975C GC-MS system equipped with a HP-5 ms GC column $(30 \mathrm{~m} \times 0.25 \mathrm{~mm} \times 0.5 \mu \mathrm{m}$; Agilent, USA) and a triple-Axis detector. The GC-MS temperature program encompassed an initial temperature of $45^{\circ} \mathrm{C}$ for $1 \mathrm{~min}$ and a ramp of $10^{\circ} \mathrm{C} / \mathrm{min}$ to $220^{\circ} \mathrm{C}$, which was maintained for $5 \mathrm{~min}$. Helium was used as the mobile phase at a flow rate of $1.0 \mathrm{~mL} / \mathrm{min}$. The injection port, interface, and MS source temperatures were 250,300 , and $180{ }^{\circ} \mathrm{C}$, respectively [29]. A reference standard comprising authentic geranyl acetate purchased from Sigma Aldrich, USA, was used for quantification.

\section{Results}

Construction of a microbial cell factory by integrating

the geranyl acetate biosynthetic pathway

into the chromosome of S. cerevisiae

As volatile esters, geranyl acetate is the essential components of fruit characteristic aroma and presents in the essential oils of various plant species. It serves as responser to stress or insect infestation. It has been reported that truncated $O$. basilicum geraniol synthase is very efficient in geraniol synthesis with geranyl diphosphate (GPP) as the substrate $[3,16]$. We found that alcohol acyltransferase gene from cultivated strawberry (SAAT) was a highly active enzyme capable of transferring the acetyl group from acetyl-CoA to various substrates, and possibly to geraniol to form geranyl acetate [29]. In order to construct a metabolic pathway for the production of geranyl acetate, the geraniol synthase (GES) gene from $O$. basilicum and SAAT were integrated into the chromosome of S. cerevisiae BY4742 at the gal80 site (Fig. 2a). The expression of the synthetic cassette was controlled by a constitutive strong promoter and the resulting strain was designated as GA01.

The production of geranyl acetate was measured by GC-MS (Fig. 3a), and the titer ranged from 0.25 to $0.63 \mathrm{mg} / \mathrm{L}$ during the fermentation process (Fig. 3b). The titer reached its maximum value after $48 \mathrm{~h}$ of cultivation, at an $\mathrm{OD}_{600}$ of 5.23. However, while the density of the yeast culture increased persistently for 5 days, geranyl acetate production did not improve accordingly, which may be due to gaseous escape of the volatile geranyl acetate during the aerobic fermentation process [32]. Besides, another four alcohol acyltransferase genes from plants were used for geranyl acetate production, but the titer was very low or no geranyl acetate was detected compared with SAAT (Additional file 1: Table S2).

\section{The expression of Erg20 mutants for improved geranyl acetate production}

GPP is the starting substrate of monoterpene production pathways [19]. However, in yeast cells GPP can be converted into FPP by the bifunctional synthase $\operatorname{Erg} 20$. In addition, since FPP is the precursor of ergosterol, the outright deletion of Erg20 is potentially lethal [5]. Accordingly, the Erg20 F96W-N127W mutant, which has a decreased FPP formation efficiency but consistent GPP production, was constructed according to an earlier report [20]. The mutated Erg20, GES and SAAT genes were integrated into the chromosome of $S$. cerevisiae BY4742 at the gal80 site (Fig. 2b). The resulting strain GA02 produced $2.64 \mathrm{mg} / \mathrm{L}$ of geranyl acetate in $48 \mathrm{~h}$, which represented a $419 \%$ increase of titer over GA01 (Fig. 4a). 

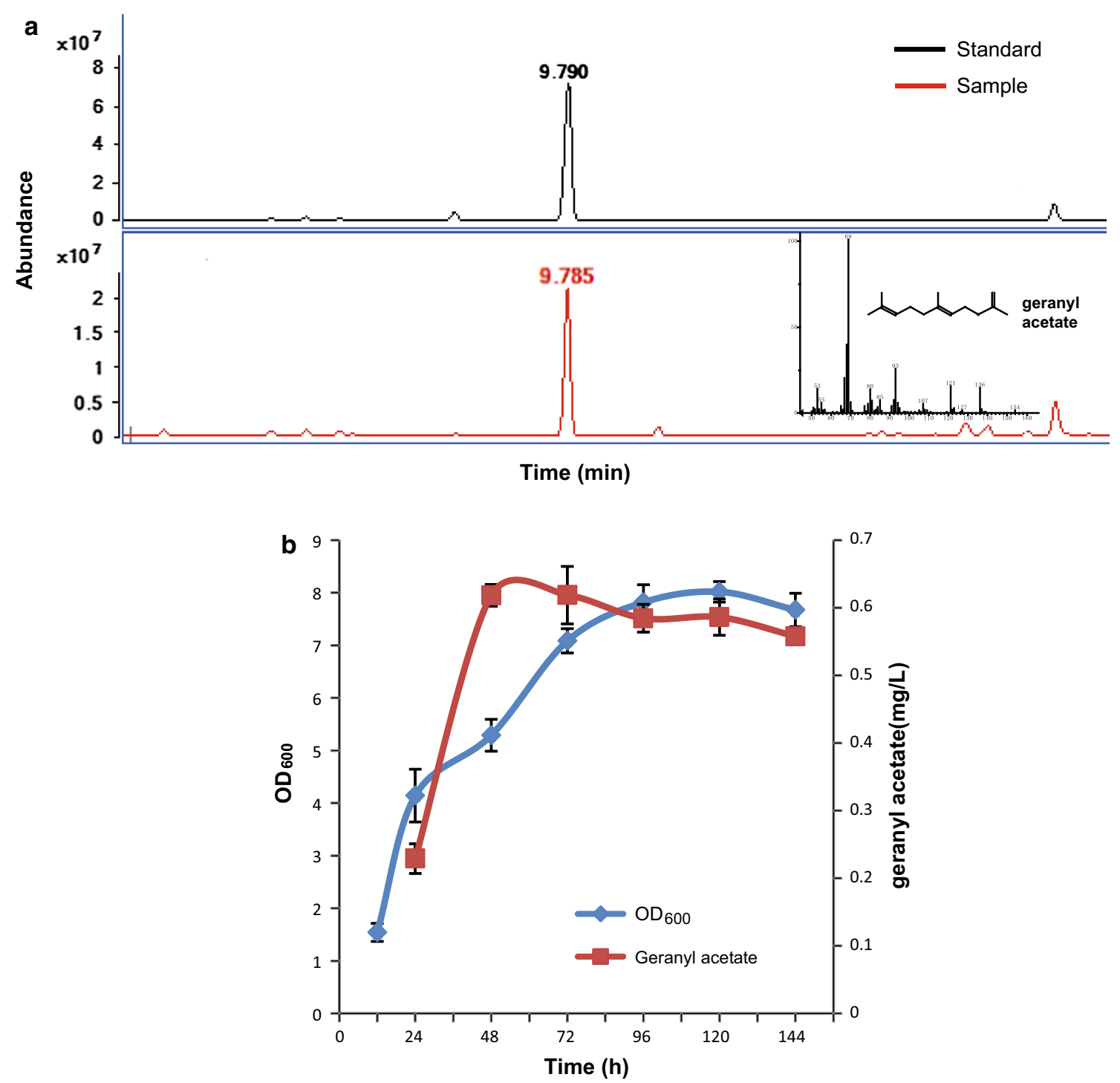

Fig. 3 Identification of fermentation products of strain $\mathrm{GA01}$. a The time-course of $\mathrm{OD}_{600}$ and geranyl acetate in aerobic fermentation of strain GA01. 38. b GC-MS analysis of a cell extract of strain GA01. The mass spectrum of geranyl acetate is shown in the top right corner and the red line indicates the authentic geranyl acetate standard

\section{Overexpression of tHMG1, IDI1 and MAF1 for the enhanced} production of geranyl acetate

Isoprenoid diphosphate isomerase (IDI1) catalyzes the isomeric interconversion between IPP and DMAPP [33], and its overexpression can therefore potentially enhance the synthesis of GPP and benefit the production of monoterpenes. MAF1 represses the transcriptional activity of RNA polymerase III, serving as a negative regulator of the biosynthesis of tRNAs [16, 18]. Since DMAPP is a common substrate of both tRNA and GPP synthesis, overexpression of MAF1 can divert the carbon flux toward GPP formation [18]. Thus, to further improve the biosynthesis of geranyl acetate, IDI1, MAF1 and tHMG1 were integrated into the chromosome of GA02, resulting in strain GA03. In this best strain, the titer of geranyl acetate reached $13.27 \mathrm{mg} / \mathrm{L}$, representing a $400 \%$ increase compared with GA02, and a remarkable $2100 \%$ increase over the starting strain GA01 (Fig. 4a).

Since $S$. cerevisiae does not have a specific GPP synthase (GPPS, EC 2.5.1.1), which belongs to the shortchain prenyltransferase family [34]. To supply more precursor for GPP synthesis, The homomeric GPPS from Arabidopsis thaliana $\left(\mathrm{GPPS}_{\mathrm{At}}\right)$ and the heteromeric GPPS from Mentha piperita $\left(\mathrm{GPPS}_{\mathrm{Mp}}\right)$ were separately 

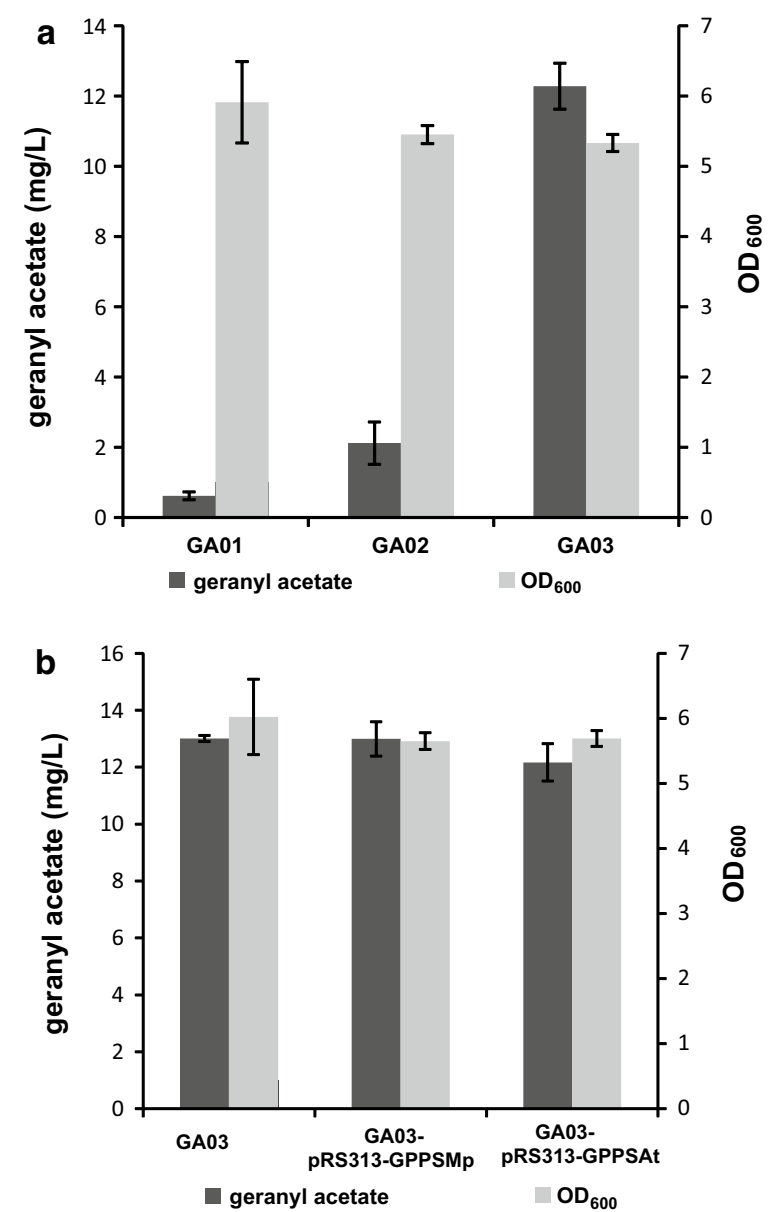

Fig. 4 Fermentation and quantification of geranyl acetate. $\mathrm{a}_{600}$ and geranyl acetate titer of the engineered strains GA01, GA02 and GA03. b Geranyl acetate titers of strains with plant GPP synthases (GPPS At: Arabidopsis thaliana GPP synthase, GPPS Mp: Mentha piperita GPP synthase)

introduced into GA03 on the plasmid pRS313-TRP, resulting in the strains GA03-pRS313-GPPS ${ }_{\mathrm{At}}$ and GA03pRS313-GPPS $_{\mathrm{Mp}}$ respectively. The relevant primers are listed in Additional file 1: Table S1, and plasmid diagrams are shown in Additional file 1: Fig. S1. Structurally, the heteromeric GPPS is composed of a large subunit (LSU) and a small subunit (SSU) [19]. The LSU is inactive alone, and the non-catalytic SSU acts as a modulator of the interaction between the two inactive subunits, resulting in an active GPPS [19]. Consequently, the flexible fusion protein linker GGGS and (GGGS) ${ }_{3}$ were introduced to construct a heteromeric Mentha piperita GPPS (LSUGGGS/(GGGS) ${ }_{3}$-SSU). Unfortunately, the introduction of plant GPPS did not effectively improve the production of geranyl acetate (Fig. 4b). Since fusion of the two subunits might affect the expression and function of
GPPS protein, we constructed another plasmid pRS313pPGK-LSU-tCYC-pTEF-SSU-tADH,the two subunit were expressed with strong promoter respectively, but the geranyl acetate production was not increased either.

\section{Improving geranyl acetate production by optimizing the fermentation conditions}

The highest geranyl acetate production of strain GA3 reached to $13.27 \mathrm{mg} / \mathrm{L}$ in SC-LEU-HIS medium after cultivated for $48 \mathrm{~h}$, and the $\mathrm{OD}_{600}$ reached about 5.23 (Fig. 4a). To further improve geranyl acetate production, we adjusted the initial $\mathrm{OD}_{600}$ of fermentation to 0.1 , which made the final $\mathrm{OD}_{600}$ increased to about 7.2 in synthetic complete drop-out medium, and to about 15.6 in YPD medium after incubated at $30{ }^{\circ} \mathrm{C}$ for $48 \mathrm{~h}$. When YPD medium was used to fermentation, the titer of geranyl acetate increased to $1.98,6.07$ and $20.48 \mathrm{mg} / \mathrm{L}$ respectively for strain GA01, GA02 and GA03 after cultivating for $48 \mathrm{~h}$ respectively, as shown in Fig. 5a. Furthermore, in order to prevent volatilization of geranyl acetate, $10 \%$ isopropyl myristate was added to the culture medium after $24 \mathrm{~h}$. The production of geranyl acetate increased to $22.49 \mathrm{mg} / \mathrm{L}$ in strain GA03 as shown in Fig. 5b, and the organic layer was easily harvested by centrifugation of the fermentation medium. However, we found geranyl acetate production did not improve continually when the strains were inoculated for 5 days. The geranyl acetate production was improved by 1.69 -fold through optimization of the fermentation conditions.

\section{Discussion}

In the past decades, more and more attention has arisen on heterologous production of the monoterpene geraniol. The highest titer of geraniol is about $2.0 \mathrm{~g} / \mathrm{L}$ in engineered $E$. coli and $1.68 \mathrm{~g} / \mathrm{L}$ in engineered $S$. cerevisiae respectively [17, 22]. However, there are rare reports for metabolic engineering of heterologous production of monoterpene esters, such as geranyl acetate. Monoterpenoids such as geranyl acetate are active compounds derived from many plants, which play important roles in protection against pathogens and attraction of animals, also traditionally used as additive of medicines, essential oils and perfume [26].

The truncated $O$. basilicum geraniol synthase (GES) and alcohol acyltransferase from strawberry (SAAT) were found with high activity in our lab [22, 29], the two genes were integrated into the chromosome of $S$. cerevisiae BY4742 at the gal80 site (Fig. 2a) and a titer of $0.63 \mathrm{mg} / \mathrm{L}$ was achieved. According to the method of Huizhou Liu's group [17], the titer of geraniol in culture medium and in yeast cells after fermentation was analyzed, which was measured to be zero. This result indicated geraniol was entirely converted to geranyl 

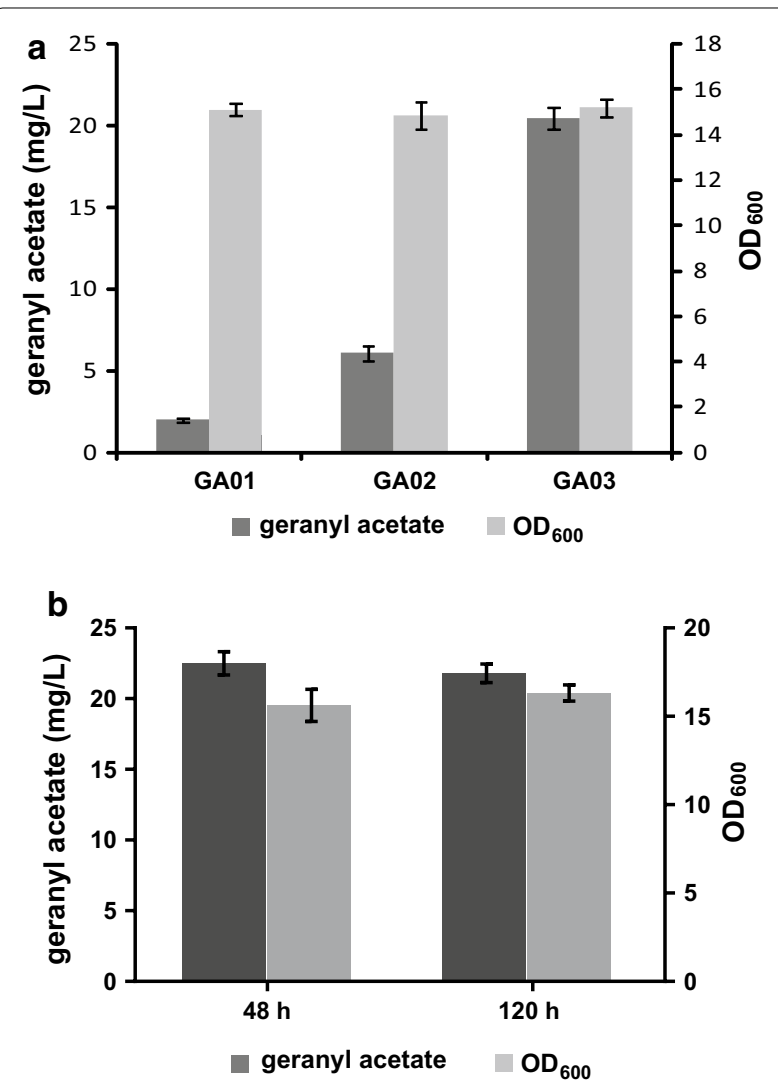

Fig. 5 Fermentation optimization and titer of geranyl acetate. a $\mathrm{OD}_{600}$ and geranyl acetate titer of the engineered strains GA01, GA02 and GA03 in YPD medium. $\mathbf{b}$ Geranyl acetate titer of strains in YPD medium with $10 \%$ isopropyl myristate added to the culture after $24 \mathrm{~h}$

acetate by SAAT by the strains we constructed, and suggested the SAAT we used was very efficient and not the rate-limiting step of the synthesis pathway. Besides, a report just published described truncated GES from Catharanthus roseus with site-directed mutation (Y436 and D501) was found to has high catalytic activity [35]. Thus, for the further improvement of the geranyl acetate production, selection and optimization of GESs might be the key focus. In engineered S. cerevisiae producing monoterpene, the farnesyl diphosphate synthase Erg20p was found to be the key enzyme that limiting monoterpene formation [20]. GPP and FPP formation are both catalyzed by Erg20p, towards either geraniol or downstream squalene synthesis. For more GPP synthesis, Erg20 F96W-N127W mutant was integrated into the genome, which had decreased farnesyl diphosphate synthase function without interference of the growth of $S$. cerevisiae. And the production of geranyl acetate increased to $2.64 \mathrm{mg} / \mathrm{L}$. Furthermore, a higher production of $13.27 \mathrm{mg} / \mathrm{L}$ was achieved by additional integration and expression of tHMG1, IDI1 and MAF1, Overexpression of which with strong promoters increased the supplement of precursors.

In the fermentation optimization process, when we used YPD media instead of the synthetic complete drop-out medium for fermentation, the $\mathrm{OD}_{600}$ value of the fermentation broth and the yield of geranyl acetate were both increased. The $\mathrm{OD}_{600}$ value could reach up to about 15.6, presenting a $198 \%$ increase compared with that in SC-LEU-HIS medium. The titer of geranyl acetate reached $20.48 \mathrm{mg} / \mathrm{L}$, showing a $54 \%$ increase compared with the production in SC-LEU-HIS medium $(13.27 \mathrm{mg} / \mathrm{L})$. So YPD was more suitable for cell growth and fermentation of the engineered strains. Besides,due to the possible gaseous escape of the volatile geranyl acetate, $10 \%$ isopropyl myristate was added to the culture after $24 \mathrm{~h}$ fermentation. TThe production of geranyl acetate increased to $22.49 \mathrm{mg} / \mathrm{L}$ in strain GA03 with YPD medium as shown in Fig. 5b. However, geranyl acetate production did not improve continually in the 5 -days fermentation process. We thought during the time, the accumulation of the product might affect the growth condition and inhibit further improvement of geranyl acetate production as described by Zhao's article [21].

To sum up, optimization of the fermentation conditions led to a 1.69-fold improvement of geranyl acetate production. And with $10 \%$ isopropyl myristate added, it might be able to prevent the volatilization of geranyl acetate and relieve the cell toxicity of geranyl acetate by extracting it from the fermentation broth [21].

\section{Conclusion}

In this research, a heterologous geranyl acetate synthesis pathway was constructed in S. cerevisiae for the first time, and a product titer of $0.63 \mathrm{mg} / \mathrm{L}$ was achieved in the starting strain. By expressing an Erg20 mutant to divert carbon flux from FPP to GPP, the geranyl acetate production was increased to $2.64 \mathrm{mg} / \mathrm{L}$, although expression of heterologous GPP did not have a significant effect. The highest production of $13.27 \mathrm{mg} / \mathrm{L}$ was achieved by additionally expressing tHMG1, IDI1 and MAF1, which represents a remarkable $2100 \%$ increase over the starting strain. Furthermore, optimization of the fermentation conditions led to $22.49 \mathrm{mg} / \mathrm{L}$ geranyl acetate production, which exhibited a 35.69 -fold increase over the parent strain GA01 (Fig. 6). To our best knowledge, this work offers the first microbial cell factory for specific production of a monoterpene ester, 


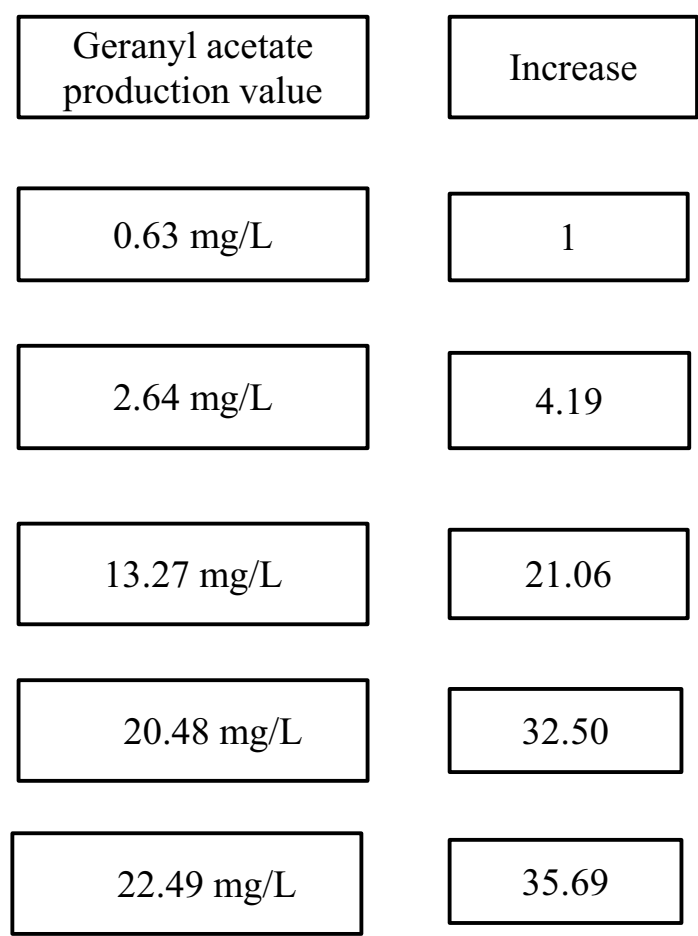

GA01

Overexpression of Erg20 mutant

GA02

Overexpression of tHMG1, IDI1 and MAF1

GA03<smiles>[3H][3H]</smiles>

GA03 in YPD medium<smiles>[CH][CH]</smiles>

GA03 in YPD medim with $10 \%$ isopropyl myristate

Fig. 6 Diagram summarizing the increase of geranyl acetate

demonstrating great potential for the heterologous production of many more economically important fragrance compounds.

\section{Additional file}

Additional file 1: Table S1. Primers used in this study. Table S2. Introduction of alcohol acyltransferases from plants and the titer of geranyl acetate. Fig. S1. Plasmid maps and DNA sequences.

\section{Authors' contributions}

TW and SWL planned and performed the experiments, analyzed and interpreted the data. TW wrote the manuscript, and SWL checked the manuscript. BZ, CB and XZ supervised the study, designed the experiments and analyzed and interpreted the results. All authors read and approved the final manuscript.

\section{Author details}

${ }^{1}$ College of Biological Sciences and Technology, Beijing Forestry University, Beijing 100083, People's Republic of China. ${ }^{2}$ Tianjin Institute of Industrial Biotechnology, Chinese Academy of Sciences, Tianjin 300308, People's Republic of China. ${ }^{3}$ Key Laboratory of Systems Microbial Biotechnology, Chinese Academy of Sciences, Tianjin 300308, People's Republic of China.

\section{Acknowledgements}

Not applicable.

\section{Competing interests}

The authors declare that they have no competing interests.

\section{Availability of data and materials}

We provide all necessary data for the publication of the article. All additional data is present in the article and the supplemental material documents.
Specifically, plasmid maps and DNA sequence data can be found in Additional file 1.

\section{Consent for publication}

I hereby give the Journal of Microbial Cell Factories the right and permission to publish this article.

\section{Ethical approval and consent to participate}

Not applicable.

\section{Funding}

This research was supported by grants from the National Natural Science Foundation of China (31522002), Natural Science Foundation of Tianjin (15JCYBJC49400), and the Tianjin Key Technology R\&D program of Tianjin Municipal Science and Technology Commission (11ZCZDSY08600).

\section{Publisher's Note}

Springer Nature remains neutral with regard to jurisdictional claims in published maps and institutional affiliations.

Received: 22 December 2017 Accepted: 11 May 2018

Published online: 05 June 2018

\section{References}

1. Mcgarvey DJ, Croteau R. Terpenoid metabolism. Plant Cell. 1995;7:1015-26.

2. Lapczynski A, Bhatia SP, Foxenberg RJ, Letizia CS, Api AM. Fragrance material review on geraniol. Food Chem Toxicol. 2008;46:S160.

3. Chen W, Viljoen AM. Geraniol-a review of a commercially important fragrance material. S Afr J Bot. 2010;76:796-807.

4. Renninger NS, Ryder JA, Fisher KJ: Jet fuel compositions and methods of making and using same. US; 2011. 
5. Anderson MS, Yarger JG, Burck CL, Poulter CD. Farnesyl diphosphate synthetase. Molecular cloning, sequence, and expression of an essential gene from Saccharomyces cerevisiae. J Biol Chem. 1989;264:19176.

6. Engels B, Dahm PS. Metabolic engineering of taxadiene biosynthesis in yeast as a first step towards Taxol (Paclitaxel) production. Metab Eng. 2008;10:201-6.

7. Ro DK, Paradise EM, Ouellet M, Fisher KJ, Newman KL, Ndungu JM, Ho KA, Eachus RA, Ham TS, Kirby J. Production of the antimalarial drug precursor artemisinic acid in engineered yeast. Nature. 2006;440:940.

8. Martin VJ, Pitera DJ, Withers ST, Newman JD, Keasling JD. Engineering a mevalonate pathway in Escherichia coli for production of terpenoids. Nat Biotechnol. 2003;21:796-802.

9. Li JW, Vederas JC. Drug discovery and natural products: end of era or an endless frontier? Science. 2009;325:161-5.

10. Jia M, Becher D, Lubuta P, Dany S, Tusch K, Schewe H, Buchhaupt M, Schrader J. De novo production of the monoterpenoid geranic acid by metabolically engineered Pseudomonas putida. Microb Cell Fact. 2014;13:1-11.

11. Carter OA, Peters RJ, Croteau R. Monoterpene biosynthesis pathway construction in Escherichia coli. Phytochemistry. 2003;64:425-33.

12. Ajikumar PK, Tyo K, Carlsen S, Mucha O, Phon TH, Stephanopoulos G. Terpenoids: opportunities for biosynthesis of natural product drugs using engineered microorganisms. Mol Pharm. 2008;5:167-90.

13. Alonso-Gutierrez J, Chan R, Batth TS, Adams PD, Keasling JD, Petzold CJ, Lee TS. Metabolic engineering of Escherichia coli for limonene and perillyl alcohol production. Metab Eng. 2013;19:33.

14. Keasling JD. Manufacturing molecules through metabolic engineering. Science. 2010;330:1355-8.

15. Amiri P, Shahpiri A, Asadollahi MA, Momenbeik F, Partow S. Metabolic engineering of Saccharomyces cerevisiae for linalool production. Biotech Lett. 2016;38:503-8.

16. Zhang W. Overproduction of geraniol by enhanced precursor supply in Saccharomyces cerevisiae. J Biotechnol. 2013;168:446

17. Liu W, Xu X, Zhang R, Cheng T, Cao Y, Li X, Guo J, Liu H, Xian M. EngineeringEscherichia colifor high-yield geraniol production with biotransformation of geranyl acetate to geraniol under fed-batch culture. Biotechnol Biofuels. 2016;9:131.

18. Fischer MJ, Meyer S, Claudel P, Bergdoll M, Karst F. Metabolic engineering of monoterpene synthesis in yeast. Biotechnol Bioeng. 2011;108:1883-92.

19. Wang G, Dixon RA. Heterodimeric geranyl(geranyl)diphosphate synthase from hop (Humulus lupulus) and the evolution of monoterpene biosynthesis. Proc Natl Acad Sci USA. 2009;106:9914-9.

20. Ignea C, Pontini M, Maffei ME, Makris AM, Kampranis SC. Engineering monoterpene production in yeast using a synthetic dominant negative geranyl diphosphate synthase. Acs Synth Biol. 2014;3:298.

21. Zhao J, Bao X, Chen L, Yu S, Jin H. Improving monoterpene geraniol production through geranyl diphosphate synthesis regulation in Saccharomyces cerevisiae. Appl Microbiol Biotechnol. 2016;100:4561-71.
22. Zhao J, Chen L, Yan Z, Yu S, Jin H, Bao X. Dynamic control of ERG20 expression combined with minimized endogenous downstream metabolism contributes to the improvement of geraniol production in Saccharomyces cerevisiae. Microb Cell Fact. 2017;16:17.

23. Asadollahi MA, Maury J, Schalk M, Clark A, Nielsen J. Enhancement of farnesyl diphosphate pool as direct precursor of sesquiterpenes through metabolic engineering of the mevalonate pathway in Saccharomyces cerevisiae. Biotechnol Bioeng. 2010;106:86-96.

24. Scalcinati G, Knuf C, Partow S, Chen Y, Maury J, Schalk M, Daviet L, Nielsen $\mathrm{J}$, Siewers V. Dynamic control of gene expression in Saccharomyces cerevisiae engineered for the production of plant sesquitepene a-santalene in a fed-batch mode. Metab Eng. 2012;14:91-103.

25. Dai Z, Liu Y, Zhang X, Shi M, Wang B, Wang D, Huang L, Zhang X. Metabolic engineering of Saccharomyces cerevisiae for production of ginsenosides. Metab Eng. 2013;20:146-56.

26. Quintansjúnior L, Moreira JC, Pasquali MA, Rabie SM, Pires AS, Schröder R, Rabelo TK, Santos JP, Lima PS, Cavalcanti SC. Antinociceptive activity and redox profile of the monoterpenes $(+)$-camphene, $p$-cymene, and geranyl acetate in experimental models. Isrn Toxicol. 2013;2013:459530.

27. Shalit M, Guterman I, Volpin H, Bar E, Tamari T, Menda N, Adam Z, Zamir $D$, Vainstein A, Weiss D, et al. Volatile ester formation in roses. Identification of an acetyl-coenzyme A. Geraniol/Citronellol acetyltransferase in developing rose petals. Plant Physiol. 2003;131:1868-76.

28. Shiota H. New esteric components in the volatiles of banana fruit (MusaSapientum L). J Agric Food Chem. 1993;41:2056-62.

29. Beekwilder J, Alvarez-Huerta M, Neef E, Verstappen FW, Bouwmeester HJ, Aharoni A. Functional characterization of enzymes forming volatile esters from strawberry and banana. Plant Physiol. 2004;135:1865-78.

30. Shao Z, Zhao H, Zhao H. DNA assembler, an in vivo genetic method for rapid construction of biochemical pathways. Nucleic Acids Res. 2009;37:e16.

31. Shao Z, Luo Y, Zhao H. DNA assembler method for construction of zeaxanthin-producing strains of Saccharomyces cerevisiae. Methods Mol Biol. 2012;898:251.

32. Brennan TC, Turner CD, Krömer JO, Nielsen LK. Alleviating monoterpene toxicity using a two-phase extractive fermentation for the bioproduction of jet fuel mixtures in Saccharomyces cerevisiae. Biotechnol Bioeng. 2012;109:2513-22.

33. Withers ST, Keasling JD. Biosynthesis and engineering of isoprenoid small molecules. Appl Microbiol Biotechnol. 2007;73:980-90.

34. Liang PH, Ko TP, Wang AH. Structure, mechanism and function of prenyltransferases. Eur J Biochem. 2002;269:3339-54.

35. Jiang GZ, Yao MD, Wang Y, Zhou L, Song TQ, Liu H, Xiao WH, Yuan YJ. Manipulation of GES and ERG20 for geraniol overproduction in Saccharomyces cerevisiae. Metab Eng. 2017;41:57-66.

\footnotetext{
Ready to submit your research? Choose BMC and benefit from:

- fast, convenient online submission

- thorough peer review by experienced researchers in your field

- rapid publication on acceptance

- support for research data, including large and complex data types

- gold Open Access which fosters wider collaboration and increased citations

- maximum visibility for your research: over $100 \mathrm{M}$ website views per year
}

At BMC, research is always in progress.

Learn more biomedcentral.com/submissions 